\title{
Different linkages in the long and short regions of the genomes of duck enteritis virus Clone-03 and VAC Strains
}

\author{
Xiaoli Liu, Zongxi Han, Yuhao Shao, Yang Li, Huixin Li, Xiangang Kong* and Shengwang Liu*
}

\begin{abstract}
Background: Duck enteritis virus (DEV) is an unassigned member in the family Herpesviridae. To demonstrate further the evolutionary position of DEV in the family Herpesviridae, we have described a 42,897-bp fragment. We demonstrated novel genomic organization at one end of the long $(L)$ region and in the entire short $(S)$ region in the Clone-03 strain of DEV.

Results: A 42,897-bp fragment located downstream of the LOFR11 gene was amplified from the Clone-03 strain of DEV by using 'targeted gene walking PCR'. Twenty-two open reading frames (ORFs) were predicted and determined in the following order: 5'-LORF11-RLORF1-ORF1-ICP4-S1-S2-US1-US10-SORF3-US2-MDVO91.5-like-US3-US4US5-US6-US7-US8-ORFX-US1-S2-S1-ICP4-3'. This was different from that of the published VAC strain, both in the linkage of the L region and $S$ region, and in the length of the US10 and US7 proteins. The MDVO91.5-like gene, ORFX gene, S1 gene and S2 gene were first observed in the DEV genome. The lengths of DEV US10 and US7 were determined to be 311 and 371 amino acids, respectively, in the Clone-03 strain of DEV, and these were different from those of other strains. The comparison of genomic organization in the fragment studied herein with those of other herpesviruses showed that DEV possesses some unique characteristics, such as the duplicated US1 at each end of the US region, and the US5, which showed no homology with those of other herpesviruses. In addition, the results of phylogenetic analysis of ORFs in the represented fragment indicated that DEV is closest to its counterparts VZV (Varicellovirus) and other avian herpesviruses.

Conclusion: The molecular characteristics of the 42,897-bp fragment of Clone-03 have been found to be different from those of the VAC strain. The phylogenetic analysis of genes in this region showed that DEV should be a separate member of the subfamily Alphaherpesvirinae.
\end{abstract}

\section{Background}

Herpesviruses are among the most persistent of all pathogens because they have coevolved with their hosts over a long period of time, and they are relatively harmless in immunocompetent hosts [1]. The family Herpesviridae comprises approximately 100 members; these viruses infect a range of host species from humans and other mammals to birds, amphibians, and reptiles [2]. On the basis of differences in cellular tropism, genome organization, and gene content, herpesviruses have been grouped into three subfamilies: Alphaherpesvirinae $(\alpha-)$,

\footnotetext{
* Correspondence: xgkong@hvri.ac.cn; swliu@hvri.ac.cn Division of Avian Infectious Diseases, National Key Laboratory of Veterinary Biotechnology, Harbin Veterinary Research Institute, the Chinese Academy of Agricultural Sciences, Harbin 150001, the People's Republic of China
}

Betaherpesvirinae $(\beta-)$, and Gammaherpesvirinae $(\gamma-)$ $[3,4]$. Currently, duck enteritis virus (DEV), also known as duck plague virus (DPV) and duck herpesvirus-1 [4], is an unassigned member of the family Herpesviridae [5].

Herpesviruses are enveloped viruses with a virion size over $100 \mathrm{~nm}$ [1]. The genomes of these viruses are linear, double-stranded DNA, and they differ in size, sequence arrangements, and base composition [2]. They also vary significantly with respect to the presence and arrangement of inverted and directly repeated sequences [6]. Herpesvirus genomes differ in the arrangement of direct and inverted repeat regions with respect to unique regions. Six types of genome structures have been confirmed adequately in herpesviruses, which are designated by letters from A to F. The A type structure

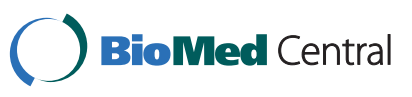

() 2011 Liu et al; licensee BioMed Central Ltd. This is an Open Access article distributed under the terms of the Creative Commons Attribution License (http://creativecommons.org/licenses/by/2.0), which permits unrestricted use, distribution, and reproduction in any medium, provided the original work is properly cited. 
consists of a unique region flanked by a direct terminal repeat at the genome ends. Type B genomes contain variable numbers of a TR (terminal reiterations) at each end of the genome. In the $\mathrm{C}$ type genome, the number of direct terminal reiterations is small but sequences longer than $100 \mathrm{bp}$ are directly repeated and subdivide the unique sequence of the genome into several well delineated stretches. The D type genome just has the repeated sequences at one terminus and in an inverted orientation internally. In the E group, the genome is divided into unique long (UL) and unique short (US) regions; each unique region is flanked by the inverted repeats. The sequences at the two termini of the $\mathrm{F}$ group are not identical and are not repeated directly or in an inverted orientation. It has been reported that DEV also contains linear, double-stranded DNA, and its genome was shown to be approximately $180 \mathrm{~kb}$ in size, with a $G$ plus C content of 64.3\% [7]. Genomic sequences of DEV have been reported recently by several Chinese research groups; however, discrepancies were found among these reports [8-18]. Genes in the UL region of DEV and their arrangement have been reported by our laboratory, and the results generally showed more similarity with Mardiviruses [8-13]. Another report showed that the LORF11 gene of the VAC strain is located at the leftmost end of the DEV genome, and that the LORF11 gene encoded a putative protein of 275 amino acids in the VAC strain [14]; both of these results differ from our previous results [12]. Meanwhile, several genes in the US region have also been reported [15-18]; however, the length of the putative proteins encoded by the US10 gene and US7 gene has been debated. In this study, we present a fragment of $42,897 \mathrm{bp}$, which contains one end of the $\mathrm{L}$ region that includes part of the LORF11 gene, which was absent from the published VAC strain, and the whole of the DEV $\mathrm{S}$ region. In addition, we demonstrated a different genomic organization of the junction of the $\mathrm{L}$ region and the $\mathrm{S}$ region in this study. These results will provide a useful comparative dataset for the study of related genes in DEV and other herpesviruses.

\section{Results}

The features of the overall sequences and determination of ORFs

A fragment of 42,897 bp downstream of the LORF11 gene was amplified from the genome of the Clone-03 strain of DEV in this study. The genome structure and the gene layout of this fragment are depicted in Figure 1. The fragment contained part of the sequence of the LORF11 gene [12], the rightmost part of the L region, the US region and its flanking sequences, and inverted repeats of the short region (IRS and TRS). The L region and IRS were interrupted by a set of tandem repeat sequences designated as $\alpha$-type-like sequences [13], as in the case of the two regions in herpes simplex virus (HSV) [19]. Another $\alpha$-type-like sequence was also found at the end of the TRS in the DEV genome. The overall G plus $\mathrm{C}$ ratio of the region sequenced was $46.09 \%$.

Twenty-two ORFs that contained more than 75 amino acids were found in the present study, which were in the order: 5'-LORF11-RLORF1-ORF1-ICP4-S1-S2-US1US10-SORF3-US2-MDV091.5-like-US3-US4-US5-US6US7-US8-ORFX-US1-S2-S1-ICP4-3'. These ORFs were predicted to encode 17 putative proteins, with the exception of LORF11, because genes in the IRS and TRS were inverted and encoded the same proteins. The start locations of all ORFs were assumed to be the first possible ATG. The motifs of each ORF are listed in Table 1.

\section{The confirmation of the junction between the $L$ region and the $\mathrm{S}$ region}

Owing to the different linkages of the $\mathrm{L}$ region and $\mathrm{S}$ region found in the genome sequences of the published DEV VAC strain [14] and our above-described sequence in the Clone-03 strain of DEV, a pair of specific primers was designed to confirm the junction of the L region and $\mathrm{S}$ region in the DEV genome. The forward primer, L25, was located in the LORF11 gene (GenBank no. EU294364), which is a gene in the DEV UL region that had only one copy in the genome compared with the genomes of other alphaherpesviruses. The reverse primer, L26, was located in the SORF3 gene, which is a gene in the US region of the DEV genome that also has a single copy in the DEV genome. The PCR product was used as the model for the second nested PCR after dilution to 1 in 1,000 . We obtained four different fragments (Figure 1), and they were $4,553 \mathrm{bp}, 4,689 \mathrm{bp}$, $4,743 \mathrm{bp}$, and $5,547 \mathrm{bp}$ in length, respectively. The results of sequencing of the four fragments showed that they were parallel with the sequences obtained using 'targeted gene walking PCR'. Consequently, we determined that the linkage between the $\mathrm{L}$ region and the $\mathrm{S}$ region should be in the following order: $5^{\prime}-L O R F 11$ RLORF1-ORF1-ICP4 -S1-S2-US1-US10-SORF3-3'.

\section{A 207-bp insertion in both the IRS and the TRS regions was not found in their counterparts in the DEV VAC strain}

In addition to the linkage of the $\mathrm{L}$ region and the $\mathrm{S}$ region, two insertions of $207 \mathrm{bp}$ were found in the presented fragment in both the IRS region and the TRS region (Figure 2), when compared with the published VAC genome. The 158-bp sequence at the 3' end of the 207-bp sequence of the IRS region was complemented with a fragment of the same length at the 5 ' end of the 207-bp sequence of the TRS region. The remaining 49-bp fragment in each of the insertions was dissociated 


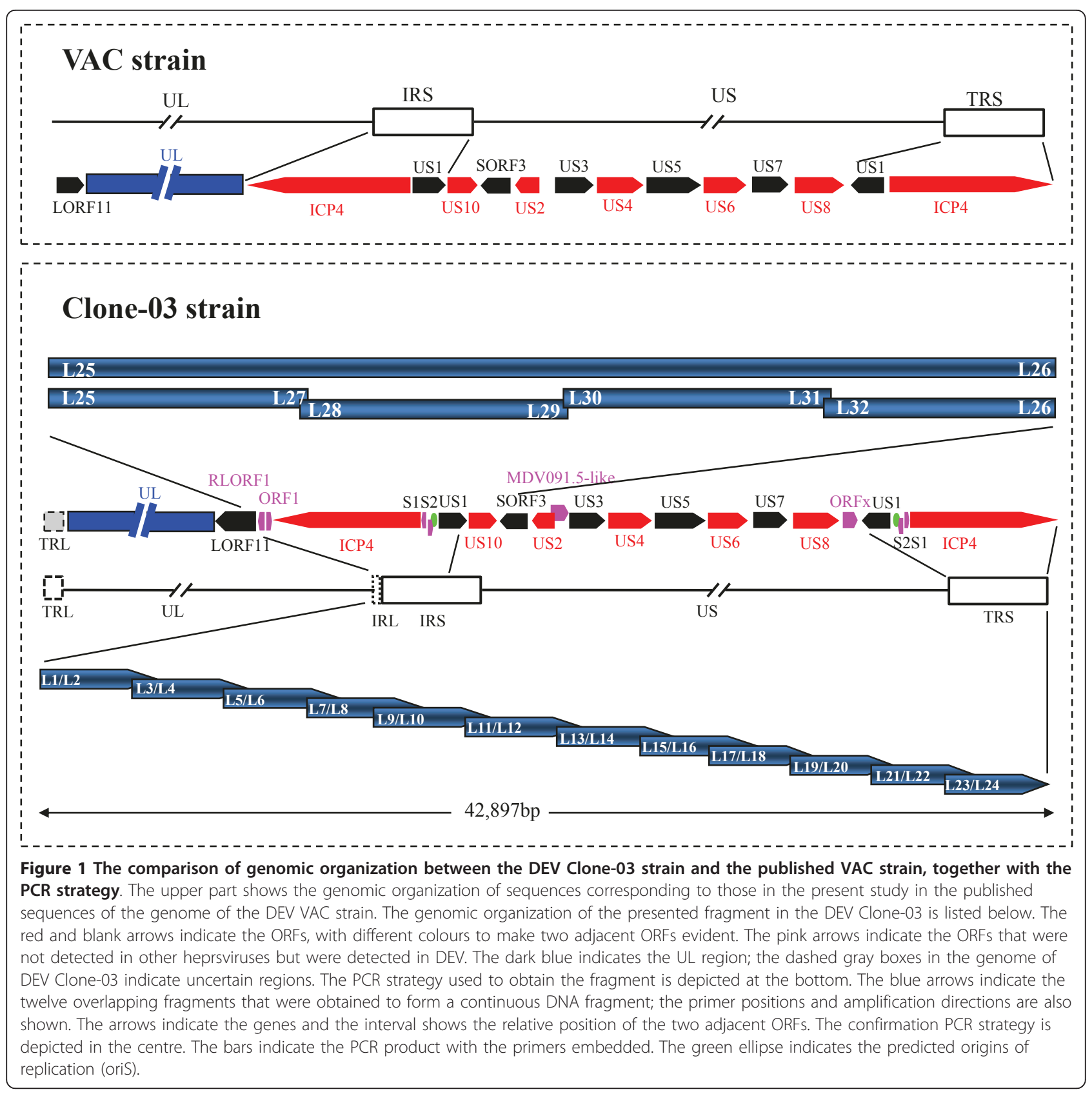

and not complemented. Both of the fragments were rich in A plus $\mathrm{T}$, with a content of $67.15 \%$.

The characteristics of new ORFs detected in the fragment Two ORFs, designated RLORF1 and ORF1, were detected in the region upstream of the $S$ region. Another copy of ORF1 was found to the left of the DEV $\mathrm{L}$ region of the genome [13]. The RLORF1 and ORF1 encoded two putative proteins of 109 and 81 amino acids, respectively. Four phosphorylation sites were predicted in the sequence of RLORF1.
In addition, eight ORFs encoding four different putative proteins (S1, S2, ICP4, and US1) in the RS region were detected. Of these proteins, S1 and S2 were identified for the first time in the present study. The $S 1$ gene encoded a putative protein of 92 amino acids, and four phosphorylation sites were predicted. No homologue of S1 was found in the proteins encoded by other herpesviruses. Another unique gene in the RS region was $S 2$, which encoded a putative protein of 96 amino acids that contained just six phosphorylation sites. ICP4 and US1 were the same as previously described $[13,14]$. 
Table 1 Core promoters searched in the neural network and polyadenylation signals predicted by POLYADQ

\begin{tabular}{|c|c|c|c|c|c|c|c|}
\hline Gene & Promoter location $^{a}$ & Promoter score & TATA sequence & TATA location & Poly(A) sequence & Poly(A) location & Poly(A) score \\
\hline RLORF1 & $N P^{b}$ & NP & NP & NP & NP & NP & NP \\
\hline ORF1 & $5,564-5,613$ & 0.93 & ATATAAAGCGGTAGT & $5,575-5,589$ & NP & NP & NP \\
\hline ICP4 & $10,928-10,977 r^{c}$ & 0.88 & TTGTAAAAT & $10,960-10,969 r$ & AATAAA & $5,867-5,872 r$ & 0.317475 \\
\hline S1 & $13,570-13,619 r$ & 0.85 & CTATCTAAGGCGACC & $13,602-13,611 r$ & NP & NP & $N P$ \\
\hline S2 & $14,725-14,774$ & 1.00 & $N P$ & NP & NP & $N P$ & NP \\
\hline US1 & $15,606-15,655$ & 0.99 & GCCTAAAAAGCACCG & $15,613-15,628$ & AATAAA & $17,015-17,020$ & 0.644949 \\
\hline US10 & $17,003-17,052 r$ & 0.94 & CAATAAACACCGCTT & $17,014-17,028$ & NP & NP & NP \\
\hline SORF3 & $19,280-19,329 r$ & 0.99 & GCTITAAAAG & $19,313-19,322 r$ & AATAAAr & $18,248-18,253 r$ & 0.138679 \\
\hline US2 & $20,407-20,456 r$ & 0.85 & GTCTAAAAGGCAGAG & $20,434-20,448 r$ & NP & NP & NP \\
\hline MDV091.5-like & NP & NP & NP & NP & NP & NP & NP \\
\hline US3 & $20,299-20,348$ & 0.85 & CCCATAAATG & $20,305-20,314$ & NP & NP & NP \\
\hline US4 & $21,799-21,848$ & 0.96 & GTATAAATTAGACAA & $21,807-21,821$ & AATAAA & $23,310-23,315$ & 0.385382 \\
\hline US5 & $23,335-23,384$ & 0.88 & GTCTTGTGTTTATAT & $23,335-23,349$ & AATAAA & $25,170-25,175$ & 0.266987 \\
\hline US6 & $25,058-25,107$ & 0.94 & CGGCAATATGTATAT & $25,062-25,076$ & NP & NP & NP \\
\hline US7 & $26,158-26,207$ & 0.92 & ATATAATTACTACGC & $26,167-26,181$ & NP & NP & NP \\
\hline US8 & NP & NP & $N P$ & NP & NP & NP & NP \\
\hline ORFx & $29,414-29,463$ & 0.93 & GTATATTAGGCCGAC & $29,421-29,435$ & NP & NP & NP \\
\hline US1 & $31,386-31,435 r$ & 0.99 & GCCTAAAAAGCACCG & $31,413-31,427 r$ & AATAAA & $30,021-30,026$ & 0.644949 \\
\hline$\$ 2$ & $32,267-32,316 r$ & 1.00 & $N P$ & NP & NP & NP & NP \\
\hline S1 & $33,422-33,471$ & 0.85 & CTATCTAAGGCGACC & $33,431-33,445$ & NP & NP & NP \\
\hline ICP4 & $36,064-36,113$ & 0.88 & TाTGTAAAAT & $36,072-36,081$ & AATAAA & $41,170-41,175$ & 0.317475 \\
\hline
\end{tabular}

${ }^{a}$ the position is according to the sequence of the whole fragment of $42,897 \mathrm{bp}$.

b NP indicates no prediction.

${ }^{c} r$ indicates reverse direction.

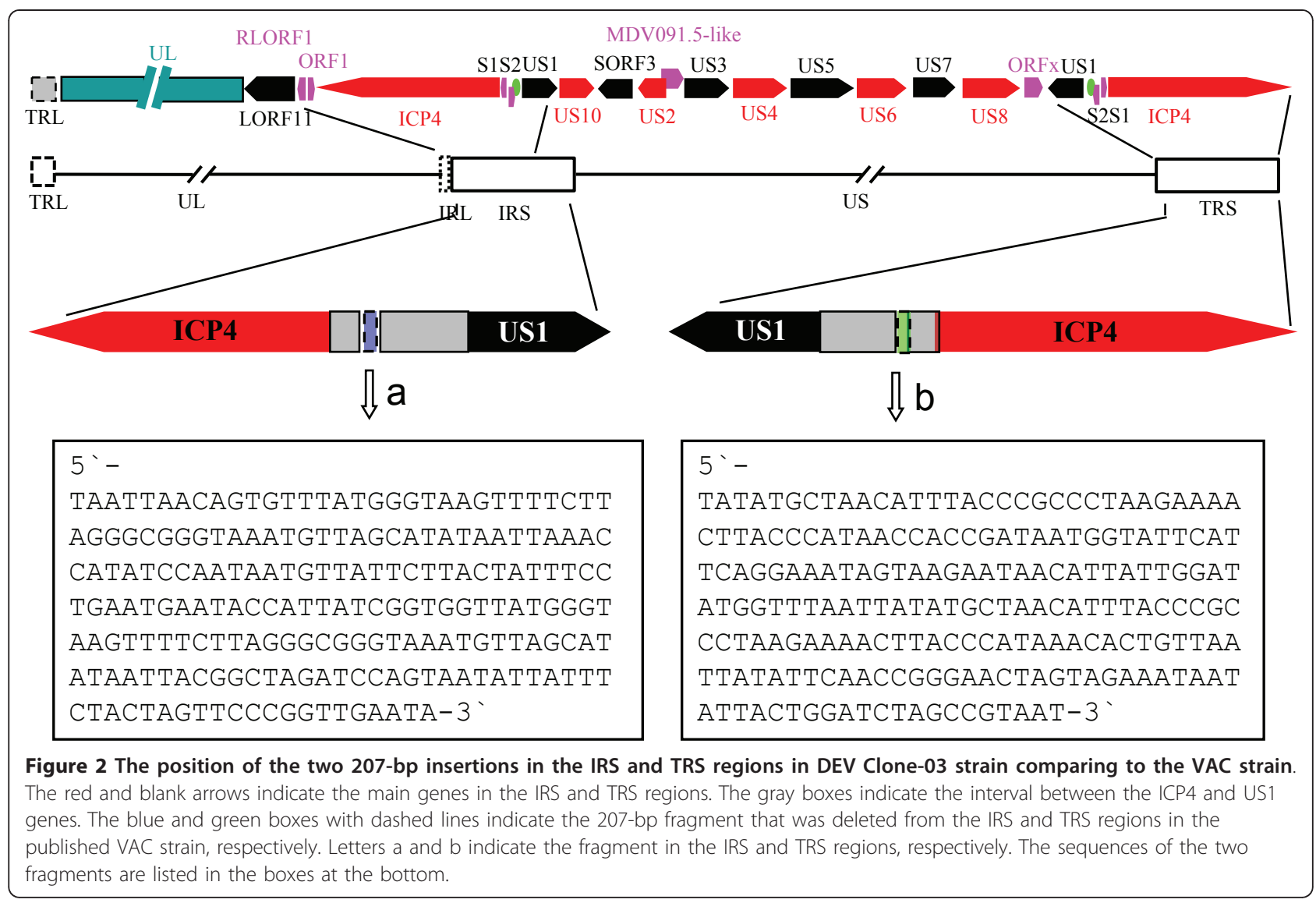


The DEV US region contained 11 ORFs that were likely to code for 11 proteins (Figure 1), which included homologues of the HSV-1 genes US10, US2, US3, US4, US6, US7 and US8 [20]. Interestingly, a unique ORF in the DEV US region, located downstream of $U S 8$, was predicted in the present study and named ORFx. The ORFx encoded a putative peptide of 118 amino acids. One transmembrane domain was detected in the ORFx between residue positions 95 and 115 at the N-terminus. Remarkably, the length of our DEV US10 was 311 amino acids, which was different from published results of 168, 169 and 298 amino acids $[14,15,17]$. We also found a sequence of 13 amino acids, CSFWCCLGHAATC (Additional file 1, Figure S1), which mapped to amino acids 236-248 and conformed to the $\mathrm{C}-\mathrm{C}-\mathrm{H}-\mathrm{C}$ zinc finger motif as described in equine herpesvirus-1 (EHV-1) [21,22]

A new gene was predicted in this study, which was $327 \mathrm{bp}$ in length and overlapped $197 \mathrm{bp}$ at the 3'-terminus of the US2 gene. It was homologous to the proteins encoded by Marek's disease virus-1 (MDV-1), MDV-2 and HVT and was designated MDV091.5-like gene. BLAST searches using the amino acid sequence showed that this protein had some amino acid similarity with putative nucleotide-binding oligomerization domaincontaining protein 2 of Gasterostrus aculeatus, the putative lyase of Rhodococcus erythropolis, and bacterial valyl-tRNA synthetase.

The transmembrane regions of the proteins encoded by the genes in the presented fragment are depicted in
Figure 3. The conserved domains of US1, SORF3, US2, US3, US4, US6, US7, US8 proteins are shown in Additional file 2, Figure S2, Additional file 3, Figure S3, Additional file 4, Figure S4, Additional file 5, Figure S5, Additional file 6, Figure S6, Additional file 7, Figure S7, Additional file 8, Figure S8, Additional file 9, Figure S9, Additional file 10, Figure S10, respectively.

\section{Phylogenetic analysis}

Phylogenetic rooted trees were constructed from alignments of the putative proteins with their homologues in other alphaherpesviruses and are shown in Figure 4 and 5. The DEV US2 gene, US3 gene, US6 gene, US7 gene and US10 gene showed closer relationships with members of Mardivirus. However, US1 showed a closer relationship between DEV and members of Simplexvirus and Varicellovirus. The DEV US4 gene showed more similarity with infectious laryngotracheitis virus (ILTV), and both clustered into the subfamily Varicellovirus (Figure 4). The DEV US8 gene fell into an outgroup position with respect to members of subfamily Alphaherpesvirinae (Figure 5), which implies that a recombination event may have occurred during the origin and evolution of the virus.

The comparison of gene layouts in the US region of DEV with those in other alphaherpesviruses

A comparison of the genetic organization of selected alphaherpesvirus US segment genes is presented in
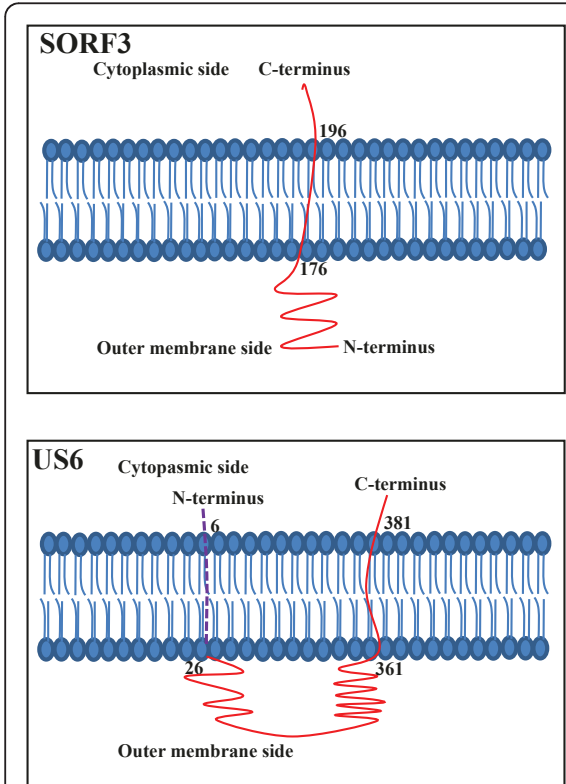
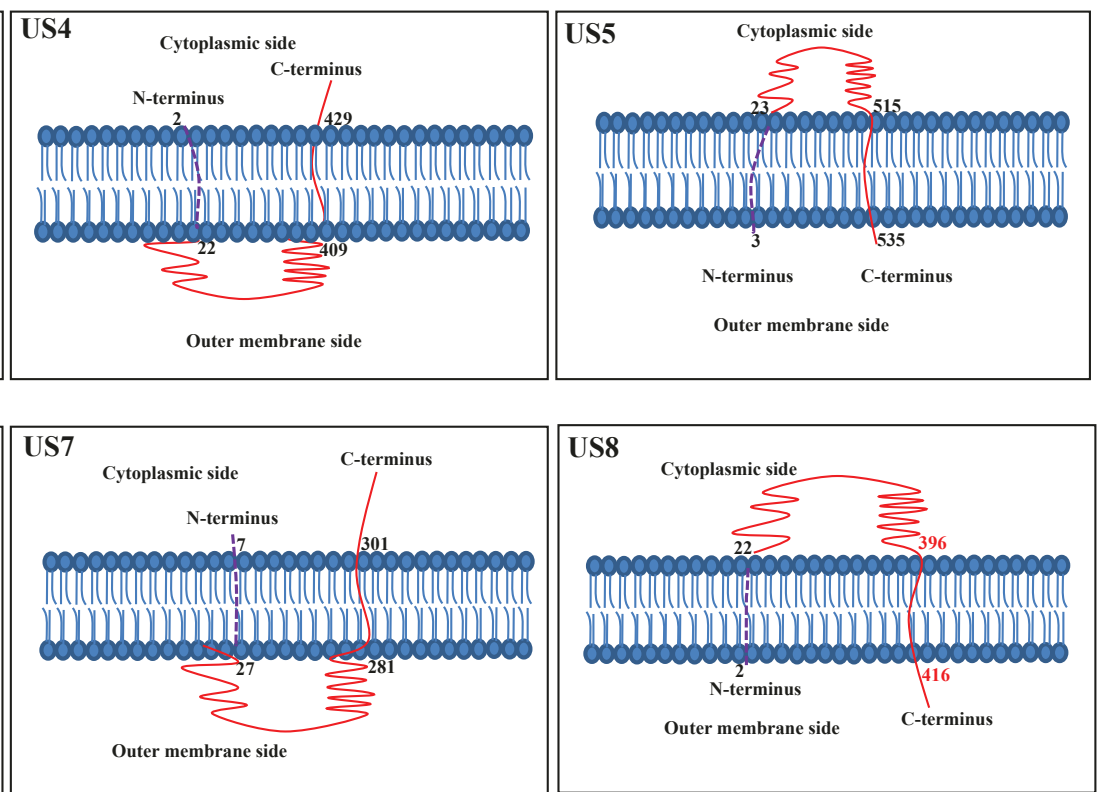

Figure 3 Transmembrane regions in the proteins encoded by DEV genes in the present fragment. The TMpred server was used to predict transmembrane regions. The dashed purple lines indicate the amino acid sequence of a potential signal peptide that may be cleaved during the maturation of proteins. 


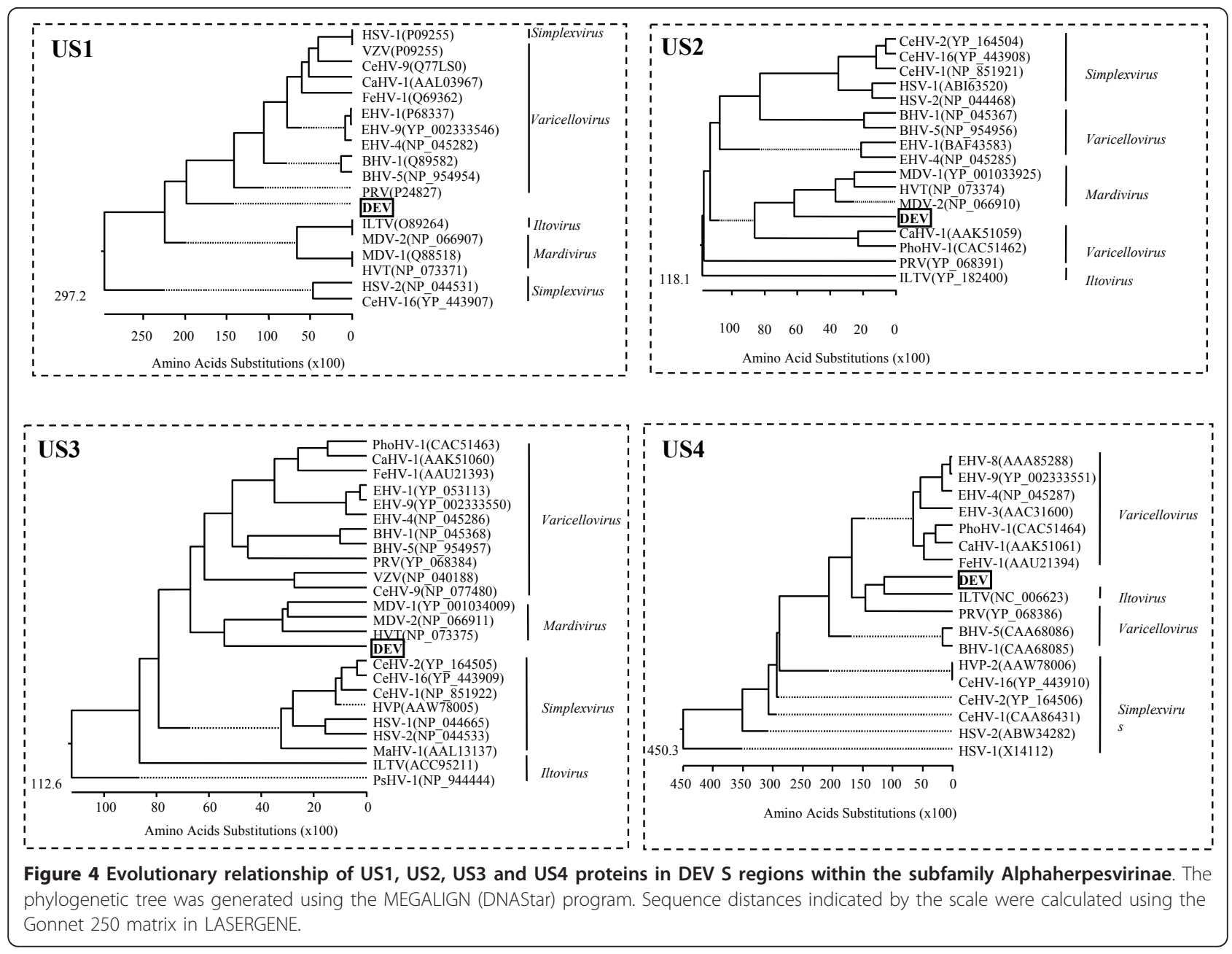

Figure 6. Despite obvious similarities, there were marked differences in gene content, organization and localization between DEV and other alphaherpesviruses. Nevertheless, these overall gene layouts are consistent with a model that accounts for the divergence of alphaherpesvirus from a common ancestor by a number of homologous and semihomologous recombination events, which resulted in concomitant loss or gain of US genes [23].

\section{Origins of replication in the $\mathrm{S}$ region}

Two well-defined origins of replication were found in the IRS and TRS of the DEV genome, designated oriS. The two oriS were palindromic structures and contained the same sequence features: two inverted 9-bp sequences, which were identical to that recognized by the origin-binding protein (OBP) encoded by the $U L 9$ binding sequence (GTTCGCAC), separated by a 43-bp AT-rich spacer sequence $(76.75 \% \mathrm{~A}+\mathrm{T})$ (Figure 7$)$. The features were the same as described for PRV (Pseudorabies virus) [24] and equine herpesvirus-1 (EHV-1) [25].

\section{Discussion}

Our laboratory has been engaged for many years in analyzing the genome sequences of DEV [8-13]. After we had completed the genome sequence of DEV Clone-03, a DEV VAC genome sequence was also published by other researchers [14]. However, some differences were detected by comparison of parts of our DEV Clone-03 strain with those of the DEV VAC strain. Herein, we presented the sequence of a 42,897-bp fragment anchored in the LORF11 gene of the DEV genome which was located at the rightward end of the UL region [12], by using the method of 'targeted gene walking PCR' (Figure 1). Comparison of the sequence of the fragment with that of the DEV VAC strain showed that our Clone-03 strain of DEV had a different gene order from that of the DEV VAC strain in this region. Consequently, we designed an additional four pairs of primers according to the new sequences and confirmed the result using nested PCR (Figure 1). The two methods obtained the same sequences, and it was demonstrated 


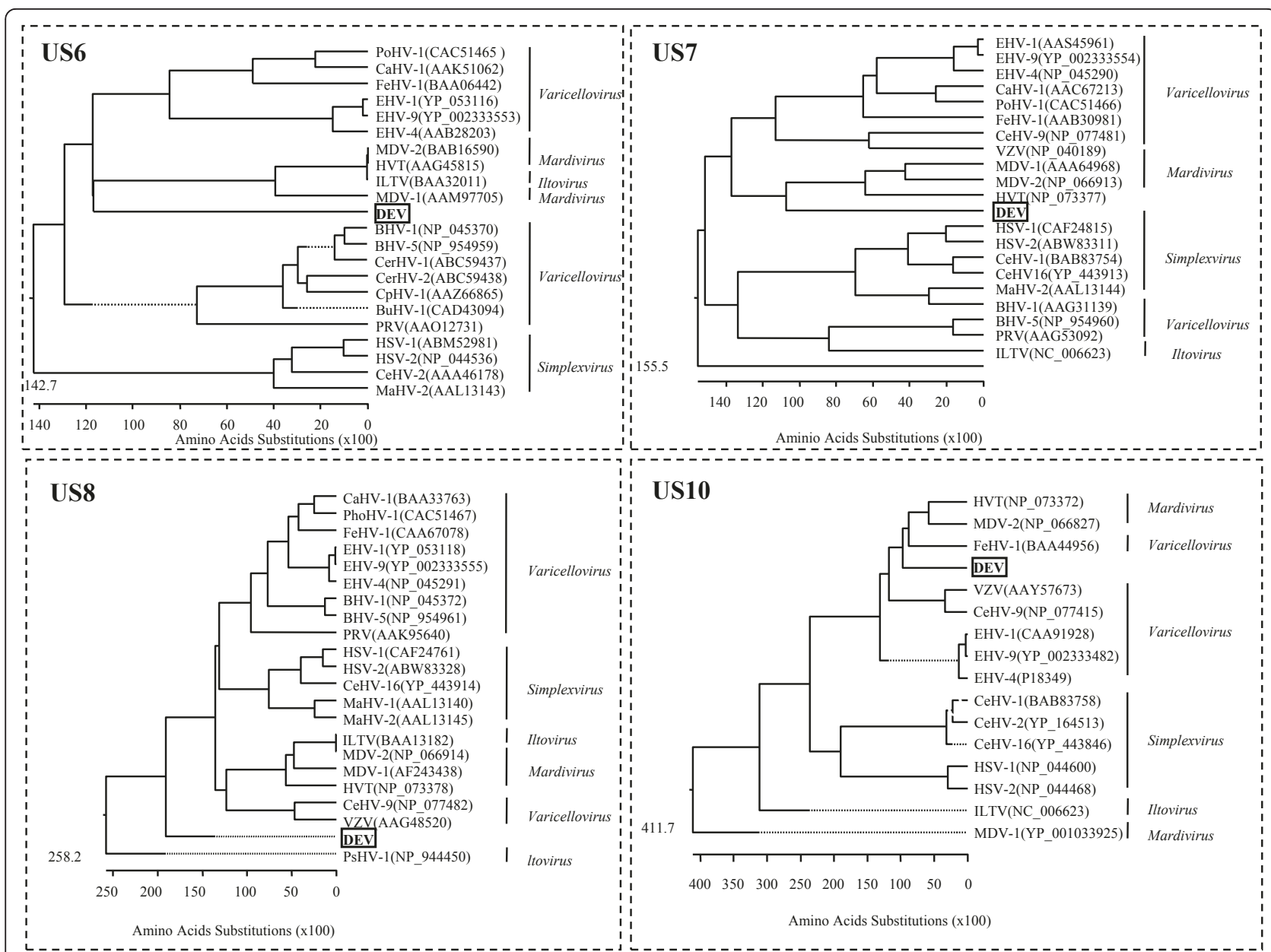

Figure 5 Evolutionary relationship of US6, US7, US8 and US10 proteins in DEV S regions within the subfamily Alphaherpesvirinae. The phylogenetic tree was generated using the MEGALIGN (DNAStar) program. Sequence distances indicated by the scale were calculated using the Gonnet 250 matrix in LASERGENE.

that the genes in this region should be in the following order: 5'-LORF11-RLORF1-ORF1-ICP4-S1-S2-US1-US10SORF3-3', which is different from the DEV VAC strain, in which the gene order is 5'-LORF11-UL-ICP4-US1US10-SORF3-3' [14]. The different linkage pattern between DEV Clone-03 and the VAC strain in the L region and $\mathrm{S}$ region is difficult to explain and requires further investigation, although a different linkage between the L and S regions of HSV was also observed between wild-type virus and cell-adapted virus [26,27].

Interestingly, we also found some novel characteristics of the sequences in the $\mathrm{S}$ region of the Clone-03 strain of DEV. Two insertions of $207 \mathrm{bp}$ in the IRS and TRS regions were found in the DEV Clone-03 strain that were absent from the VAC strain. It has been reported that some fragments were lost during serial passage of MDV [28]. Hence, we speculated that the insertion of the two 207-bp fragments in the DEV Clone-03 strain and their absence from the VAC strain might be due to the different passage levels [28]. The $S 1$ gene, $S 2$ gene, RLORF1 gene, ORF1 gene and ORFx gene that were observed in the Clone-03 strain in this study also had similar sequences in the VAC genome; however, those genes showed no homologues in other alphaherpesviruses. Those genes may be potential markers to differentiate DEV from other alphaherpesviruses.

Davison and McGeoch concluded that differences in gene layout in the S component between HSV-1 and VZV have resulted from expansion and contraction of IRS/TRS during evolution [23]. This may also be the case for the DEV genome. Unlike those of MDV-1, MDV-2 and HVT, the DEV US1 gene was duplicated and also inverted to the other end of the US, as is that of PRV [24]. Similarly, the presence of two copies of the US1 gene in DEV does not imply that the virus expresses two forms of ICP22 [24]. Although the pattern of the two copies of the US1 gene in the DEV genome showed a similar gene layout to those of PRV, the existence of the 


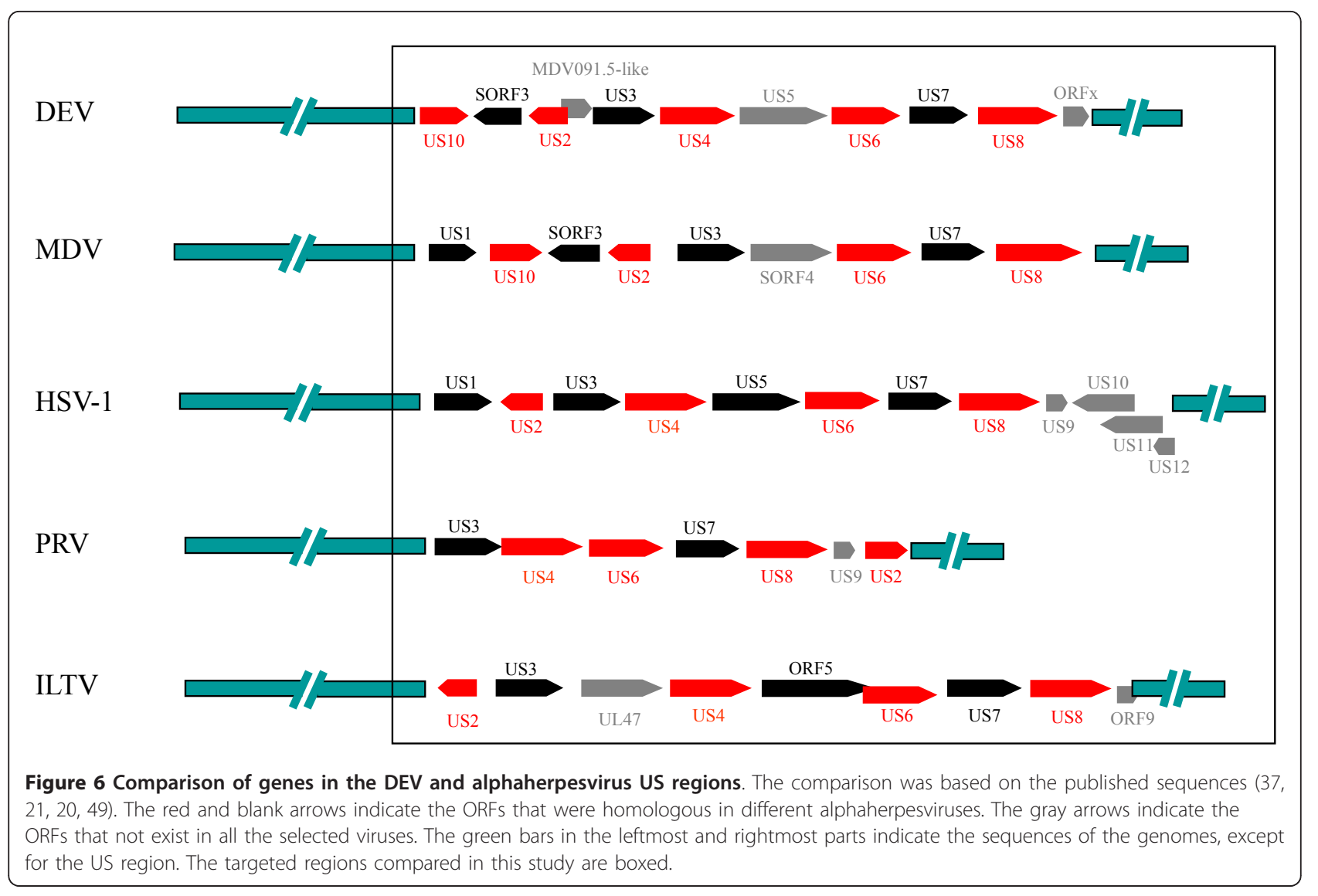

LORF11 gene at the rightward end of the UL region indicated that the organization of the DEV genome may be similar to that of other avian herpesviruses. The presence of the SORF3 gene and the MDV091.5-like gene, and the translocation of the US1O gene in the DEV genome, further suggests a close relationship between DEV and other avian herpesviruses. In addition, the phylogenetic analysis of most genes in the presented fragment further indicated a close relationship between DEV and viruses in the subfamily Mardivirus. However, the US region of DEV contained some genes that were absent from the genomes of other avian herpesviruses, such as US4 and ORFx, which indicates that DEV may be a unique member of the subfamily Alphaherpesvirinae.

Replication of the viral genome is a central event in the life cycle of herpesviruses. The initiation of viral DNA synthesis marks the commitment of the infected cell to the production of new infectious virus and, in most instances, cell death. HSV-1 contains three origins of DNA replication of two types: one copy of oriL located at the centre of the UL region of the genome and two copies of oriS located in the repeat regions that flank the US region of the genome [29]. The reasons for the three potential origins of replication in the viral genome are not apparent in HSV. In this study, we predicted two copies of
oriS in the RS region of DEV. It has been reported that the deletion of the oriL in HSV resulted in reduced replication in mouse tissues and reduced reactivation from latent infection. Thus, oriL may be required for DNA replication in certain tissues [29]. Although oriL was absent from the DEV genome, the core sequence of oriS, which typically contains an origin recognition element and a DNAunwinding element, was unchanged [29]. This absence of oriL from DEV may be associated with the evolution of the viral genome, may lead to different characteristics of the replication of DEV from those of other herpesviruses, and may even result in functional deletions from the genome of DEV in comparison with other herpesviruses.

\section{Conclusion}

In this study, we demonstrated a different organization of genes in the rightward part of the $L$ region and the whole $\mathrm{S}$ region in the Clone-03 strain of DEV, when compared with the VAC strain. Several novel characteristics were also detected in this region that have not been reported in the VAC strain, including the presence of S1,S2, ORFX and MDV091.5-like genes and two insertions in the IRS and TRS regions. The genomic order and the characteristics of the genes in this region, together with phylogenetic analysis based on the 


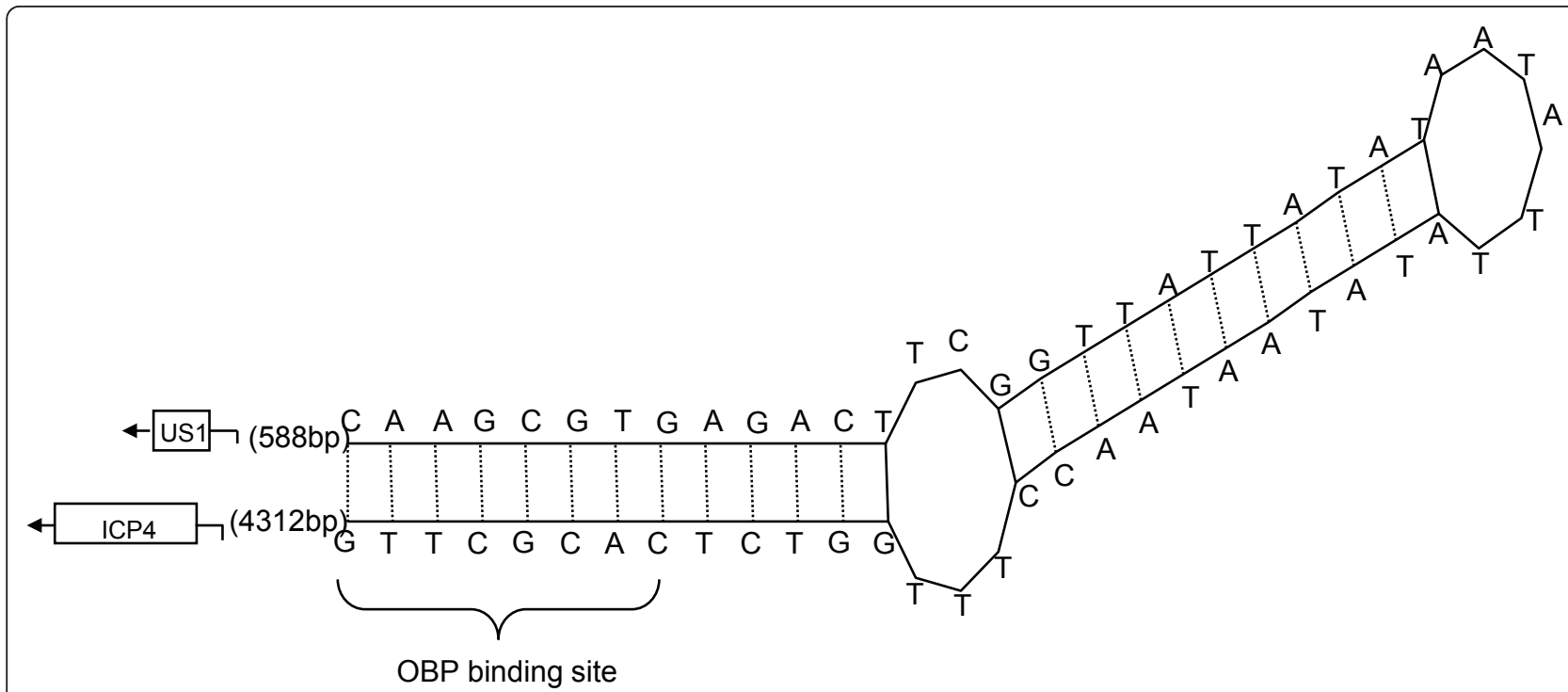

Figure 7 Hairpin-like secondary structure reflecting palindromic nature of sequences of oriS. The arrows and boxes at flanking the palindromes represented the two genes that flanked the oriS. The distances in nucleotides between the oriS sequence and the two genes are shown in brackets.

putative proteins encoded by the genes investigated in the present study showed that DEV should be a unique member of the subfamily Alphaherpesvirinae.

\section{Methods}

\section{Virus stock preparation}

The Clone-03 strain of DEV was used in this study [8-13]. The virus stocks were produced by propagation in chicken embryo fibroblasts (CEF) in Dulbecco's minimum essential medium (DMEM) with $8 \%$ fetal bovine serum. The infected CEFs were harvested when the cytopathic effect (CPE) reached 80\%. After three freezethaw cycles, the virus stocks were confirmed primarily by electron microscopy and polymerase chain reaction (PCR) as described previously [8-13].

\section{DNA extraction, polymerase chain reaction and sequencing}

The viral DNA was extracted from the virus stocks as described previously [9]. The 'targeted gene walking PCR', as described previously [30,31], was used to amplify the targeted DEV genome fragment, as illustrated in Figure 1. Briefly, four nonspecific 'walking' primers, N1, N2, N3 and $\mathrm{N} 4$ [10] were used to walk the genome of DEV. A pair of specific primers, L1 and L2, was designed on the basis of the partial sequence of DEV LORF11 published in GenBank (GenBank no. EU294364) [12]. The PCR was carried out by using L1 and L2 as forward primers; the four nonspecific primers were used as reverse primers. Finally, a 2,998-bp fragment (F1) was amplified, anchored from the DEV LORF11 gene. Targeted primer L3 and internal primer L4 were designed on the basis of the newly generated fragment, and was used to amplify the neighbouring gene fragment with one of the four nonspecific primers. Similarly, primers L5-L6, L7-L8, L9-L10, L11-L12, L13L14, L15-L16, L17-L18, L19-L20, L21-L22 and L23-L24 (Figure 1) were designed and used in the subsequent PCR amplifications. The primers used in PCR amplifications in this study are listed in Table 2.

The PCR was carried out in a $25 \mu$ l reaction volume as described previously [9]. The reaction was performed at $95^{\circ} \mathrm{C}$ for $5 \mathrm{~min}$, followed by 30 cycles of $94^{\circ} \mathrm{C}$ for 1 min, $50^{\circ} \mathrm{C}$ for $1 \mathrm{~min}$ and $72^{\circ} \mathrm{C}$ for $3 \mathrm{~min}$; the reaction was ended by elongation at $72^{\circ} \mathrm{C}$ for $10 \mathrm{~min}$. The PCR products were analyzed on a $0.8 \%$ agarose gel. The PCR products were sequenced directly or cloned into the pMD18-T vector (TaKaRa, Dalian, China) according to the manufacturer's instructions and used for sequencing. Each of the fragments was sequenced at least three times from different PCR products.

The determination of open reading frames (ORFs) in the presented fragment and genomic organization in the junction between the $L$ region and $S$ region in the DEV genome

The sequences obtained were assembled using the Gene Runner (version 3.00, Hastings Software, Inc., Hudson, NY, USA). The ORFs and genomic organization in the junction of the $\mathrm{L}$ region and $\mathrm{S}$ region, and the layout of genes in the $S$ region, were determined by comparison with the sequence counterparts of Marek's disease virus (MDV), HSV-1 and varicella-zoster virus (VZV). The 
Table 2 Sequences of oligonucleotides primers used for PCR amplification

\begin{tabular}{|c|c|c|}
\hline Primer & Sequences $\left(5^{\prime}-3^{\prime}\right)$ & Position $^{a}$ \\
\hline L1 & AGTCCAGTCATCTCCATCCG & $1,562-1,581$ \\
\hline L2 & ACGATTTGGCTGTGCTGTAG & $1,730-1,749$ \\
\hline L3 & CTGTCTTAAGGTTAGGGCTGGC & $4,532-4,553$ \\
\hline$\llcorner 4$ & GTAGGAAATATTGAGCCGAG & $4,826-4,845$ \\
\hline L5 & TTCTGATGTTTTGGCAAGCC & $7,578-7,597$ \\
\hline L6 & CAGAATGGCGCTTTGTTTGG & $7,669-7,688$ \\
\hline L7 & TTGAAGATAGGTTGCTCGTAG & $11,272-11,292$ \\
\hline L8 & ATACAGGAAAATTAACGAT & $11,367-11,385$ \\
\hline L9 & ATGTAGCAGTTTGTCAAAC & $14,193-14,212$ \\
\hline L10 & AAGATGAGTCAACACCGAAGG & $14,445-14,465$ \\
\hline L11 & TATTCCATCCAGTTGCTCCC & $17,732-17,751$ \\
\hline $\mathrm{L} 12$ & CTTGTAAAGCTGGCCGCTAC & $17,978-17,997$ \\
\hline L13 & CGATCTGCTTTCGCTTTCCG & $21,725-21,744$ \\
\hline L14 & TAGCTGGTATGGCAACAATG & $21,895-21,914$ \\
\hline L15 & GAAGTTAACGGAGGAAGTATT & $26,043-26,063$ \\
\hline L16 & GCATATAATTACTACGCAACC & $26,165-26,185$ \\
\hline L17 & GTCATCCTTGTTATGTTGA & $29,697-29,715$ \\
\hline L18 & CCTACTTGGTGGTCGGCCA & $29,795-29,813$ \\
\hline L19 & CAGGATTTGATAACTAACC & $34,266-34,284$ \\
\hline $\mathrm{L} 20$ & ATAAGCGCACTAGATGGCAG & $34,473-34,492$ \\
\hline L21 & TGAACGGACCTITGCTAATGAC & $37,793-37,813$ \\
\hline L22 & GAGGGGTGGTACTGGTTCCG & $38,120-38,139$ \\
\hline L23 & CCTACAATAACCTGGGAACT & $40,770-40,789$ \\
\hline L24 & GATCTTGTCCGATGGGGATG & $40,949-40,968$ \\
\hline L25 & ATGGGACAGTCCCTACCGTTGGCCTCGATTCAAAGCTTCTCAG & $1-43$ \\
\hline L26 & GGACTGCAGGCCTITCAACGGCCCCTGATATGGCTACTATGTC & $18,716-18,758 r^{b}$ \\
\hline L27 & GCCAGCCCTAACCTTAAGACAG & $4,532-4,553 r$ \\
\hline L28 & CAACCCCGCCCACAATAAGC & $4,043-4,062$ \\
\hline L29 & GCTTACATGCTTITCCCCGC & $8,712-8,731 r$ \\
\hline $\mathrm{L} 30$ & CGAACCGTCACAGTCTGCAG & $8,592-8,611$ \\
\hline L31 & GCCGCGCAGTAAGTACTCAG & $13,315-13,334$ \\
\hline L32 & TGCCGATATCATTGGTTCAT & $13,212-13,231$ \\
\hline N1 & TATAGGTTT(C/A)TGTT & $N P^{c}$ \\
\hline N2 & CTITTGAGCTG & NP \\
\hline N3 & GAATGTGA(A/G)AA & NP \\
\hline N4 & CATGTCTGCCGA & NP \\
\hline
\end{tabular}

\footnotetext{
a the position is according to the sequence of the whole fragment of $42,897 \mathrm{bp}$.
}

${ }^{\mathrm{b}} \mathrm{r}$ indicates the reverse direction.

${ }^{\mathrm{c}} \mathrm{NP}$ indicates no prediction.

same program was used to detect ORFs encoding proteins of greater than or equal to 75 amino acids with a methionine (M) start codon. The predicted ORFs and flanking sequences were evaluated for coding potential by detecting the promoter http://www.fruitfly.org/seq_tools/promoter.html[32], and the presence of TATA box http://motif.genome.jp/ and transcription terminal signals http://rulai.cshl.org/tools/polyadq/polyadq_form. $h t m l$. Searches of the deduced proteins for signal peptides http://www.cbs.dtu.dk/services/SignalP/, transmembrane regions http://www.ch.embnet.org/software/
TMPRED_form.html, N-linked glycosylation sites http:// www.cbs.dtu.dk/services/NetNGlyc/ and serine, threonine and tyrosine phosphorylation sites http://www.cbs. dtu.dk/services/NetPhos/ were also performed online. The secondary structure of sequences in the oriS was constructed by using GeneQuest in DNAStar.

\section{Confirmation of the junction between the $L$ region and the $S$ region by specific PCR}

Owing to the different order of genes in the junction of the $\mathrm{L}$ region and $\mathrm{S}$ region in the DEV Clone-03 in this 
study and the reported DEV VAC strain [14], one pair of specific primers, L25 and L26 (Table 2), was designed to confirm the result. Primer L25 was located within the LORF11 gene and L26 was located within the SORF3 gene. This pair of primers was used in the first nested PCR. Other primers, L27, L28-L29, L30-L31, and L32 (Table 2), were also used in the second nested PCR. The position of the primers and the strategy for confirmation of the sequence are shown in Figure 1.

The PCR was carried out in a $25 \mu \mathrm{l}$ reaction volume. The first nest of the PCR reaction was performed at $95^{\circ}$ $\mathrm{C}$ for $5 \mathrm{~min}$, followed by 35 cycles of $94^{\circ} \mathrm{C}$ for $1 \mathrm{~min}$, $50^{\circ} \mathrm{C}$ for $1 \mathrm{~min}$ and $72^{\circ} \mathrm{C}$ for $8 \mathrm{~min}$; the reaction was ended by elongation at $72^{\circ} \mathrm{C}$ for $10 \mathrm{~min}$. The PCR product was analyzed on a $0.8 \%$ agarose gel and was used as the template for the second nest. The second nested PCR was performed at $95^{\circ} \mathrm{C}$ for $5 \mathrm{~min}$, followed by 30 cycles of $94^{\circ} \mathrm{C}$ for $1 \mathrm{~min}, 53^{\circ} \mathrm{C}$ for $1 \mathrm{~min}$ and $72^{\circ} \mathrm{C}$ for 3 min; the reaction was ended by elongation at $72^{\circ} \mathrm{C}$ for $10 \mathrm{~min}$. The products of the second nested PCR were cloned and sequenced, respectively.

\section{Phylogenetic analysis}

Homologue searches were conducted using BLAST searching [33] and phylogenetic analysis was performed using the MEGALIGN program in Lasergene (DNAStar) with CLUSTAL W multiple alignment and weight matrix Gonnet 250 [13]. The result was confirmed by use of the MAGE package (Version 4.0). The sequences of the herpesviruses that were used as reference strains for homology analysis were obtained from the GenBank database and the GenBank accession numbers are given in the phylogenetic trees.

\section{GenBank accession numbers}

The DNA sequence of 42,897 bp from the DEV Clone03 genome has been deposited in the GenBank database with the GenBank accession no. HQ009801.

\section{Additional material}

Additional file 1: Figure S1: Multiple alignments of homologues based on US10 proteins of DEV Clone-03 and other typical strains of the subfamily Alphaherpesvirinae. The pink box indicate the probable C-C-H-C zinc finger motif in US10 proteins by comparison with their homologues in other herpesviruses.

Additional file 2: Figure S2: Multiple alignments of homologues based on US1 proteins of DEV Clone- 03 and other typical strains of the subfamily Alphaherpesvirinae.

Additional file 3: Figure S3: Multiple alignments of homologues based on SORF3 proteins of DEV Clone- 03 and other avian herpesviruses

Additional file 4: Figure S4: Multiple alignments of homologues based on US2 proteins of DEV Clone- 03 and other typical strains of the subfamily Alphaherpesvirinae. The conserved domains were indicated by pink boxes.
Additional file 5: Figure S5: Multiple alignments of homologues based on the amino acid sequences in the $\mathrm{N}$-terminus of US3 proteins of DEV Clone- 03 and other typical strains of the subfamily Alphaherpesvirinae. The conserved domains (from I to VI) were indicated by pink boxes.

Additional file 6: Figure S6: Multiple alignments of homologues based on the amino acid sequences in the C-terminus of US3 proteins of DEV Clone- 03 and other typical strains of the subfamily Alphaherpesvirinae. The conserved domains (from VII to XI) were indicated by pink boxes.

Additional file 7: Figure S7: Multiple alignments of homologues based on US4 proteins of DEV Clone- 03 and other typical strains of the subfamily Alphaherpesvirinae. The conserved domains were indicated by pink boxes.

Additional file 8: Figure S8: Multiple alignments of homologues based on US6 proteins of DEV Clone- 03 and other typical strains of the subfamily Alphaherpesvirinae. The conserved domains were indicated by pink boxes.

Additional file 9: Figure S9: Multiple alignments of homologues based on US7 proteins of DEV Clone-03 and other typical strains of the subfamily Alphaherpesvirinae.

Additional file 10: Figure S10: Multiple alignments of homologues based on US8 proteins of DEV Clone- 03 and other typical strains of the subfamily Alphaherpesvirinae. The conserved domains were indicated by pink boxes.

List of abbreviations used

DEV: duck enteritis virus; DVE: duck viral enteritis; DPV: duck plaque virus; ORF: open reading frame; L: long; S: short; UL: unique long; US: unique short; a: Alphaherpesvirinae; $\beta$ : Betaherpesvirinae; $\gamma$ : Gammaherpesvirinae; CEF: chicken embryo fibroblasts; DMEM: Dulbecco's minimum essential medium; CPE: cytopathic effect; PCR: polymerase chain reaction; MDV: marek's disease virus; HVT: turkey herpesvirus; VZV: varicella-zoster virus; PRV: pseudorabies virus; M: methionine; ILTV: infectious laryngotracheitis; EHV: equine herpesvirus; CeHV: cercopithecine herpesvirus; CaHV-1: canid herpesvirus-1.

\section{Authors' contributions}

$X L, S L$ and $X K$ designed research; $X L, Z H, Y S$ and $Y L$ performed research; $X L$, $\mathrm{SL}, \mathrm{XK}$ and $\mathrm{HL}$ analyzed data; and XL, SL and XK wrote the paper. All authors read and approved the final manuscript.

Competing interests

The authors declare that they have no competing interests.

Received: 14 October 2010 Accepted: 2 May 2011

Published: 2 May 2011

References

1. E Gershburg, JS Pagano, Conserved herpesvirus protein kinases. Biochim Biophys Acta. 1784, 203-212 (2008)

2. JP Weir, Genomic organization and evolution of the human herpesviruses. Virus Genes. 16, 85-93 (1998). doi:10.1023/A:1007905910939

3. B Roizman, LE Carmichael, F Deinhardt, G De-The, AJ Nahmias, W Plowright, F Rapp, P Sheldrick, M Takahashi, K Wolf, Herpesviridae. Definition, provisional nomenclature, and taxonomy. The herpesvirus study group, the international committee on taxonomy of viruses. Intervirology. 16, 201-217 (1981). doi:10.1159/000149269

4. B Roizmann, RC Desrosiers, B Fleckenstein, C Lopez, AC Minson, MJ Studdert, The family Herpesviridae: an update. Arch Virol. 123, 425-449 (1992). doi:10.1007/BF01317276

5. AJ Davison, R Eberle, B Ehlers, GS Hayward, DJ McGeoch, AC Minson, PE Pellett, B Roizman, MJ Studdert, E Thiry, The order Herpesvirales. Arch Virol. 154, 171-177 (2009). doi:10.1007/s00705-008-0278-4

6. B Roizman, The human herpesviruses in The herpesviridae, a brief introduction. in Fields of Virology, ed. by Roizman B, Whitley RJ, Lopez C (New York: Raven Press, 1993), pp. 1-9 
7. R Gardner, J Wilkerson, JC Johnson, Molecular characterization of the DNA of anatid herpesvirus 1. Intervirology. 36, 99-112 (1993)

8. H Li, S Liu, Z Han, Y Shao, S Chen, X Kong, Comparative analysis of the genes UL1 through UL7 of the duck enteritis virus and other herpesviruses of the subfamily alphaherpesvirinae. Genet Mol Bio. 32, 121-128 (2009). doi:10.1590/\$1415-47572009005000003

9. H Li, S Liu, X Kong, Characterization of the genes encoding UL24, TK and $\mathrm{gH}$ proteins from duck enteritis virus (DEV): a proof for the classification of DEV. Virus Genes. 33, 221-227 (2006). doi:10.1007/s11262-005-0060-6

10. S Liu, S Chen, H Li, X Kong, Molecular characterization of the herpes simplex virus 1 (HSV-1) homologues, UL25 to UL30, in duck enteritis virus (DEV). Gene. 401, 88-96 (2007). doi:10.1016/j.gene.2007.06.022

11. R An, H Li, Z Han, Y Shao, S Liu, X Kong, The UL31 to UL35 gene sequences of duck enteritis virus correspond to their homologs in herpes simplex virus 1. Acta Virol. 52, 23-30 (2008)

12. SW Liu, HX Li, Y Li, ZX Han, YH Shao, R An, XG Kong, Pylogeny of duck enteritis virus: Evolutionary relationship in the Family Herpesviridae. Intervirology. 51, 151-165 (2008). doi:10.1159/000146865

13. XL Liu, SW Liu, HX Li, ZX Han, YH Shao, XG Kong, Unique sequence characteristics of genes in the leftmost region of unique long region in duck enteritis virus. Intervirology. 52, 291-300 (2009). doi:10.1159/000235742

14. YF Li, B Huang, XL Ma, J Wu, F Li, W Ai, MX Song, HC Yang, Molecular characterization of the genome of duck enteritis virus. Virology. 391 151-152 (2009). doi:10.1016/j.virol.2009.06.018

15. Y Zhao, JW Wang, F Liu, B Ma, Molecular analysis of US10, S3, and US2 in duck enteritis virus. Virus Genes. 38, 243-248 (2009). doi:10.1007/s11262008-0315-0

16. Y Zhao, JW Wang, B Ma, FY Liu, Molecular analysis of duck enteritis virus US3, US4 and US5 gene. Virus Genes. 38, 289-294 (2009). doi:10.1007/ s11262-008-0326- $x$

17. $\mathrm{Y} \mathrm{Hu}, \mathrm{H}$ Zhou, Z Yu, H Chen, M Jin, Characterization of the genes encoding complete US10, SORF3, and US2 proteins from duck enteritis virus. Virus Genes. 38, 295-301 (2009). doi:10.1007/s11262-009-0329-2

18. Y Zhao, JW Wang, Characterization of duck enteritis virus US6, US7 and US8 gene. Intervirology. 53, 141-145 (2010). doi:10.1159/000274974

19. A Dolan, FE Jamieson, C Cunningham, BC Barnett, DJ McGeoch, The genome sequence of herpes simplex virus type 2. J Virol. 72, 2010-2021 (1998)

20. DJ McGeoch, A Donald, FJ Rixon, Sequence determination and genetic content of the short unique region in the genome of herpes simplex virus type 1. J Mol Biol. 181, 1-13 (1985). doi:10.1016/0022-2836(85)90320-1

21. MF Summers, Zinc fingers motif for single stranded mucleic acids? Investigatjions by nuclear magnetic resonance. J Cell Biochem. 45, 41-48 (1991). doi:10.1002/jcb.240450110

22. VR Holden, RR Yaamanchili, RN Harty, DJ O' Callaghan, Identification and characterization of an equine herpesvirus 1 late gene encoding a potential zinc finger. Virology. 188, 704-713 (1992). doi:10.1016/0042-6822(92)90525-T

23. AJ Davison, DJ McGeoch, Evolutionary comparisons of the $S$ segments in the genomes of herpes simplex virus type 1 and varicella-zoster virus. J Gen Virol. 67, 597-611 (1986). doi:10.1099/0022-1317-67-4-597

24. BG Klupp, CJ Hengartner, TC Mettenleiter, LW Enquist, Complete, annotated sequence of the Pseudorabies virus genome. J Virol. 78, 424-440 (2003)

25. EAR Telford, MS Watson, K McBride, AJ Davison, The DNA sequence of Equine herpesvirus-1. Virology. 189, 304-316 (1992). doi:10.1016/0042-6822 (92)90706-U

26. H Delius, JB Clements, A partial denaturation map of herpes simplex virus type 1 DNA: evidence for inversions of the unique DNA regions. J Gen Virol. 33, 125-133 (1976). doi:10.1099/0022-1317-33-1-125

27. GS Hayward, RJ Jacob, SC Wadsworth, Anatomy of herpes simplex virus DNA: evidence for four populations of molecules that differ in the relative orientations of their long and short components. Proc Natl Acad Sci USA. 72, 4243-4247 (1975). doi:10.1073/pnas.72.11.4243

28. K Hirai, K Ikuta, S Kato, Structural changes of the DNA of Marek's disease virus during serial passage in culture cells. Virology. 115, 385-389 (1981). doi:10.1016/0042-6822(81)90119-7

29. JW Balliet, PA Schaffer, Point mutation in herpes simplex virus type 1 oriL, but not in oris, reduce pathogenesis during acute infection of mice and impair reactivation from latency. J Virol. 80, 440-450 (2006). doi:10.1128/ JVI.80.1.440-450.2006
30. PC Chang, ML Hsieh, JH Shien, DA Graham, MS Lee, HK Shieh, Complete nucleotide sequence of avian paramyxovirus type 6 isolated from ducks. J Gen Virol. 82, 2157-2168 (2001)

31. JD Parker, PS Rabinovitch, GC Burmer, Targeted gene walking polymerase chain reaction. Nucleic Acids Res. 19, 3055-3060 (1991). doi:10.1093/nar/ 19.11.3055

32. M Reese, NL Harris, FH Eeckman, Large scale sequencing specific neural networks for promoter and splice site recognition. in Biocomputing Proceedings of the 1996 Pacific Symposium: 2-7 Jan 1996, ed. by Hunter L, Klein TE (Singapore: World Scientific Publishing Inc, 1996)

33. SF Altschul, W Gish, W Miller, EW Mysers, DJ Lipman, Basic local alignment search tool. J Mol Bio. 215, 3389-3402 (1990)

doi:10.1186/1743-422X-8-200

Cite this article as: Liu et al: Different linkages in the long and short regions of the genomes of duck enteritis virus Clone-03 and VAC Strains. Virology Journal 2011 8:200.

\section{Submit your next manuscript to BioMed Central and take full advantage of:}

- Convenient online submission

- Thorough peer review

- No space constraints or color figure charges

- Immediate publication on acceptance

- Inclusion in PubMed, CAS, Scopus and Google Scholar

- Research which is freely available for redistribution

Submit your manuscript at www.biomedcentral.com/submit
Ciomed Central 